\title{
Dynamics of urban fire correlations with detrended fluctuation analysis
}

\author{
Jinghong Wang ${ }^{1,2}$ \\ ${ }^{1}$ State Key Laboratory of Fire Science, University of Science and Technology of China, Hefei 230026, China, \\ arain@mail.ustc.edu.cn \\ ${ }^{2}$ USTC-CityU Joint Advanced Research Centre (Suzhou), Suzhou 215123, China
}

\begin{abstract}
To explore and understand the intrinsic dynamic mechanisms of urban fire system is an important prerequisite for the fire risk analysis and management. By analyzing the time series of urban fire system, it is helpful to achieve this goal. In this paper, the detrended fluctuation analysis (DFA) is applied to study the long-range correlations of urban fires in both small and large spatial scales with a case of Chinese city. It is found that the time series of direct economic loss caused by urban fires presents stable anti-power law correlations, while the time interval series of urban fire occurrence presents persistent long-range power-law correlations with two scaling exponents. Based on the characteristics of these scaling exponents, the intrinsic dynamics of urban fires is discussed. It is found that the effect of human activities on the dynamics of urban fire system may become prevalent at small timescales, while at large timescales the effect of human activities is not obvious in influencing the long-range correlation patterns of urban fire system, which may be controlled mainly by some general characteristics of a city such as the meteo-climatic conditions.
\end{abstract}

Keywords: Urban fire, Dynamics, Detrended fluctuation analysis, Long-range correlation.

\section{基于去趋势波动分析的城市火灾相关性动态研究 王静虹 ${ }^{1,2}$}

1.中国科学技术大学火灾科学国家重点实验室, 合肥 230026

2.中科大-香港城大联合高等研究中心（苏州），苏州 215123

\begin{abstract}
摘要: 对城市火灾系统的内在动力学机制进行探讨和研究, 是城市火灾风险分析与管理的重要前提, 而对 城市火灾系统的时间序列进行分析，则有助于实现这一目标。本文运用去趋势波动方法，在不同的空间尺度上对 城市火灾发生及损失规模的时间序列进行分析，探讨了城市火灾在时间和空间上的长程相关性特征。研究发现， 城市火灾造成的直接经济损失时间序列表现出稳定的反幂律相关性，而城市火灾发生的时间间隔序列表现出稳定 的长程幂律相关性, 且这种长程相关性具有两个分段的标度指数。基于这种分段标度指数的特征, 本文进一步讨 论了城市火灾系统的内在动力学机制, 发现在较短的时间尺度上, 人类活动对城市火灾系统动态特征的影响应是 普遍存在的, 而在较长的时间尺度上, 人类活动对城市火灾系统的这种长程相关性特征并无明显的影响, 而是一 些城市的普遍特征如气象气候条件等起着主导作用。
\end{abstract}

关键词：城市火灾, 动态特征, 去趋势波动分析, 长程相关性

\section{1. 引言}

城市火灾系统即城市火灾和它的影响因素所构成
的整体, 是一个复杂系统, 涉及到人类活动、城市结构、 社会经济、气象环境等多种因素。城市火灾与这些因 素之间存在着复杂的相互作用,城市火灾事件之间也 
存在着相互关联。对城市火灾系统进行科学合理的风 险评估和预测, 是关系到城市火险控制及保障居民生 命财产安全的重要工作。作为一个复杂的非线性动力 学系统, 城市火灾系统通常以其原始的输出信号 (火 灾发生时间及规模) 表现出一种非稳定性的随机特征。 因此, 在分析城市火灾的内在动力学机制、预测未来 的发展趋势以及研究影响城市火灾发生的各种社会经 济、人类活动和环境因素之前, 必须首先找出城市火 灾在时间和空间尺度上的分布形式。已有研究发现, 城市火灾系统在时空尺度上呈现幂律分布的形式, 即 具有一种自组织临界性 (SOC) 特征 ${ }^{[1-3]}$ 。这些研究大 多基于一些大空间范围内的原始火灾数据, 例如, 在 ${ }^{[4]}$ 的研究中, 采用了中国和日本全国范围内的火灾数据 进行了火灾分布的对比研究, 其研究结果对于整体上 理解城市火灾系统的分布特征具有积极的意义。然而, 当涉及到某个具体的城市乃至城市中某个具体的区域 时, 这些研究所提供的方法并不足以帮助我们了解其 具体的火灾分布形式以及内在的动力学机制。目前, 基于社区的防火概念 (community-oriented fire protection）已经越来越受到国内外的重视 ${ }^{[5]}$, 其中的 一个重要方面就是需要在更小的空间尺度上对城市火 灾系统进行更为精细的分析, 进而更有助于掌握某些 具体城市区域的火险特征, 以及区域和整体的火灾分 布形式之间的区别和联系。

从分析方法上来说, 已有的研究通常采用传统的 功率谱方法和Hurst分析方法对城市火灾的静态数据序 列进行分析。这些方法对于序列的变化趋势和分形特 征有着较好的分析效果, 且在不考虑一个较长时间尺 度下序列背景发生变化的前提下, 其结果具有较高的 可靠性。但是城市火灾系统附属于城市系统之中, 其 时间序列本身具有很强的不稳定性, 这种不稳定性往 往与城市结构的变化、社会经济的发展等背景因素紧 密耦合, 因此采用传统的分析手段, 其结果的可靠性 将在很大程度上受到这些不稳定因素的影响, 同时也 无法反映出城市火灾系统在某一时间尺度内演化的长 程特征 ${ }^{[6]}$ 。一种优化的方法就是Peng ${ }^{[7]}$ 等人提出的去趋 势波动 (Detrended Fluctuation Analysis, 简称DFA) 分 析方法。该方法可以有效挖掘出一个看似不稳定序列 内部蕴含着的自相似性, 以及序列是否具有长程相关
性。与传统方法相比, DFA方法最大的优势在于可以 消除序列的局部趋势, 从而避免将时间序列的短程相 关、非平稳性虚假地检测为长程相关性。该方法目前 已经被广泛运用于诸多领域, 如生物医学领域中的 DNA序列研究 ${ }^{[8]}$ 、心率变异性研究 ${ }^{[9,10]}$ 、气候 ${ }^{[11,12]}$ 、经 济领域 ${ }^{[13,14]}$ 、地震 ${ }^{[15]}$ 、森林火灾 ${ }^{[16]}$ 等, 但是在城市火 灾领域尚较少见诸报导。本文运用DFA方法, 分析了 某些城市区域中火灾时空分布形式的长程趋势, 以及 这种长程趋势与城市区域特征之间的定性联系, 以期 对城市火灾系统的内在动力学机制进行一个初步的探 讨。

\section{2. 数据和方法}

本文分析的数据来源于安徽省消防总队, 是合 肥市 2000-2009 年的全部火灾数据, 数据包括起火 时间、起火场所、火灾原因及直接财产损失等。运 用 DFA 方法对合肥市全市以及不同区域的火灾时间 序列进行长程相关性分析, 同时为解释这种长程相 关性的潜在原因, 或者说动力学机制, 文中进一步 考虑了不同城市区域的特征, 如人口、社会经济活 动等因素。

DFA方法已被证明可以有效揭示时间序列的长 程相关性程度。对于一个非稳定时间序列, 应用DFA 方法进行分析的主要步骤为:

首先计算该时间序列的累积离差:

$$
y(k)=\sum_{i=1}^{k}\left[x(i)-x_{\text {ave }}\right]
$$

其中 $x_{a v e}$ 是该序列中所有数据的平均值。接着将 这个新的序列 $y(k)$ 等分成 $\mathrm{N} / \mathrm{n}$ 个具有同样长度 $\mathrm{n}$ 的区 间, 在每个区间中通过最小二乘法进行拟合, 所获 得的拟合直线斜率代表了该区间中数据的趋势。用 $y_{n}(k)$ 表示每个区间中通过拟合获得的趋势函数, 则 去趋势后的子区间时间序列为 $y(k)-y_{n}(k)$ 。最后计 算去趋势后时间序列的波动均方根:

$$
F(n)=\sqrt{\frac{1}{N} \sum_{k=1}^{N}\left[y(k)-y_{n}(k)\right]^{2}}
$$

如果每个区间中的拟合多项式 $y_{n}(k)$ 采用了 
线性、二次、三次乃至更高阶 $\mathrm{m}$ 的多项式, 那么就将 算法分别记为 $\mathrm{DFA}_{1}, \mathrm{DFA}_{2}, \mathrm{DFA}_{3}, \ldots \mathrm{DFA}_{\mathrm{m}}$ 等。显 然m阶的DFA滤去了累积离差中的 $m$ 阶趋势成分以 及原始序列中的 $\mathrm{m}-1$ 阶趋势成分。本文采用DFA3进 行数据的分析, 可以消除序列中的线性、二阶和三 阶趋势。 $\mathrm{n}$ 的取值范围与原始序列的长度 $\mathrm{N}$ 有关, 初 始值 (最小值) 设置均为 4 , 此时的区间数 ( $\mathrm{N} / \mathrm{n}$ ) 最多; 将希望得到的区间数最小值设置为 4 , 此时对 应的n值即为最大值。根据这个算法, 就可以获得 $F(n)$ 和 $n$ 之间的关系。

在不同的时间尺度（不同的区间长度 $n$ ) 上重复 这样的算法, 就可以获得平均波动程度 $F(n)$ 与时间尺 度 $n$ 之间的关系。通常 $F(n)$ 会随着时间尺度 $n$ 的增加而 递增。如果在双对数坐标中 $F(n)$ 与 $n$ 存在幂律关系 (自 相似性), 即

\section{$F(n) \propto n^{\alpha}$}

此处的拟合直线斜率 $\alpha$, 就可以表征该时间序列 的标度指数，表明该时间序列中的信号并不代表纯 粹的随机过程, 而是具有一定的长程相关性特点。 不同的 $\alpha$ 值表示原始时间序列具有不同的分布形式:

当 $\alpha=0.5$ 时, 该序列即为白噪声;

当 $\alpha=1.0$ 时, 该序列即为 $1 / \mathrm{f}$ 噪声;

当 $0.5<\alpha<1$ 时, 说明该序列具有稳定的长程幂律 特征, 如果在时刻 $\mathrm{t}$ 以前存在上升(或下降)趋势, 那 么在时刻 $\mathrm{t}$ 以后总体上也存在上升(或下降)趋势, 即 过去发生的事件对未来事件的发生产生影响;

当 $0<\alpha<0.5$ 时, 该序列表现出反相关性, 或具 有非持久性;

当 $\alpha>1$ 时, 时间上的长程相关性存在, 但是不 再服从幂律分布。

\section{3. 结果和讨论}

3.1 全市火灾数据的DFA分析结果（较大空间尺度）

合肥市位于北纬 $31.52^{\circ}$ 、东经 $117.17^{\circ}$, 作为安徽省省 会, 是全省政治、经济、文化、金融和商业中心，同 时也是长江流域的一个重要城市。合肥全市下辖瑶海 区、庐阳区、蜀山区和包河区四个中心城区, 肥东县、
肥西县和长丰县三个郊县 (在本文中不作为分析对 象）, 以及高新技术产业区和经济开发区两个经济开 放区域。合肥市行政区划如图1所示。

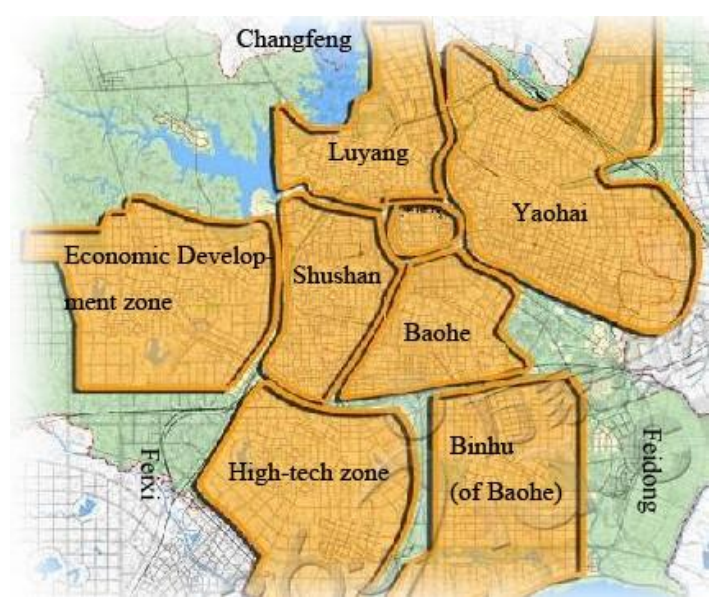

图1 合肥市行政区划示意图

图2所示为合肥市2000-2009年间共6328起火灾的 直接经济损失时间序列。为了判断城市火灾的长程相 关性是否受特殊值的影响, 比如某一年中损失数值特 别大的重特大火灾, 本文除了真实火灾序列之外, 根 据真实火灾损失数据构建了一个随机序列, 即将真实 序列中非零的数值用 $\left(0,10^{6}\right)$ 这样一个随机分布进行取 代。表1列出了合肥市2000-2009年间每年单起火灾造 成的直接经济损失前三大的数值, 可以看到仅有很少 的数据 (以下划线标出) 超出了百万元量级, 换句话 说, 百万元这个量级可以反映合肥市2000-2009年火灾 造成的直接经济损失的一个普遍最大数量级水平, 因 此将随机分布函数选择为 $\left(0,10^{6}\right)$ 。图3所示为真实序列 和随机序列的DFA分析结果。可以看到, 虽然图中两 条曲线整体形状并不完全一致, 但是拟合直线的斜率 几乎一致。真实序列和随机序列的拟合直线斜率分别 为 0.48 和 0.49 , 均小于 0.5 , 这就说明城市火灾的损失 规模具有长程时间尺度上的反相关性, 即所分析序列 具有非持久性, 过去发生的事件对未来事件具有不确 定的影响。城市火灾损失规模受多种不确定因素的影 响, 一些极端阈值的出现往往受到各种不确定因素的 作用, 这也正体现了火灾损失规模序列的非持久性特 征。但不管是真实序列还是模拟的随机序列, 这种空 
间尺度上的长程特征均比较稳定存在, 而损失规模一 定程度上反映了火灾在空间上的分布特征，由此可以 认为, 城市火灾在空间上的长程特征应是受到某种动 力学机制的作用, 即与时间上的分布形式有所联系。 因此，本文进一步对城市火灾发生的时间间隔序列进 行了去趋势波动分析, 以发掘其在时间尺度上的分布 特征。

表1. 2000-2009年合肥市每年单起火灾造成的前三大 直接经济损失 (万元)

\begin{tabular}{llll}
\hline 年份 & 最大 & 第二大 & 第三大 \\
\hline 2000 & $\underline{2178.94}$ & 64.35 & 18.98 \\
2001 & 62.06 & 18.68 & 12.00 \\
2002 & $\underline{2692.09}$ & 56.10 & 24.28 \\
2003 & 33.36 & 27.04 & 16.54 \\
2004 & 80.42 & 38.05 & 28.71 \\
2005 & 25.08 & 25.01 & 17.72 \\
2006 & 33.60 & 29.23 & 23.52 \\
2007 & $\underline{445.22}$ & 17.05 & 15.66 \\
2008 & $\underline{654.87}$ & $\underline{115.62}$ & 87.76 \\
2009 & $\underline{\mathbf{5 0 0 . 0 0}}$ & $\underline{231.90}$ & 50.00 \\
\hline
\end{tabular}

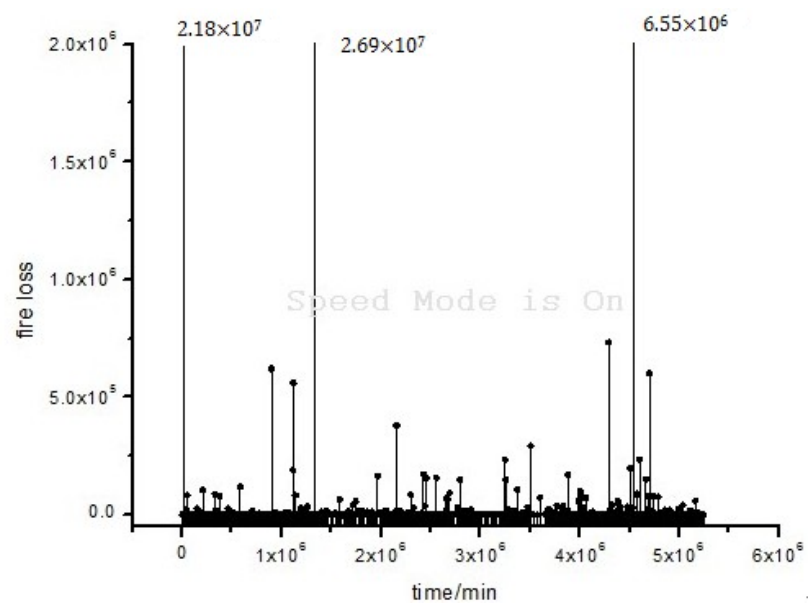

图2 合肥全市2000-2009年火灾时间序列 横轴代表每起火灾的发生时间, 单位为分钟; 坚轴 代表每起火灾造成的直接经济损失, 单位为元人民 币。

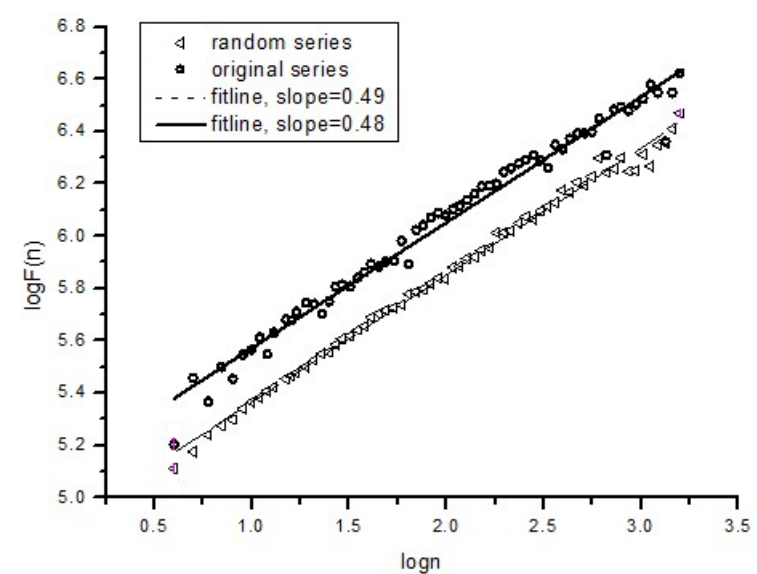

图3 合肥全市2000-2009年火灾直接经济损失时间 序列的DFA分析结果

根据合肥市2000-2009年间6328起火灾的发生时 间，可以形成一个时间间隔序列，其中的每一个数据 均代表两起时间上相邻的火灾事件的等待时间。运用 DFA方法分析这个时间间隔序列, 结果如图4所示。可 以看到, 全市火灾时间间隔序列表现出分段的标度指 数特征, 分段处约在 64 天左右的位置, 短程标度 $\alpha_{1}$ 为 0.73 , 小于长程标度 $\alpha_{2}$ 为 0.97 。与此类似, 对单个年份 的全市火灾时间间隔序列进行DFA分析, 选取2007、 2008 和 2009 三年的火灾数据进行分析, 表 2 所示为这三 年每年发生的火灾起数。图5所示为这三年火灾时间间 隔序列的标度指数特征，发现单个年份的火灾时间间 隔序列同样具有分段的标度指数特征, 分段处约在 15 天左右的位置, 如图5所示。2007年的短程标度指数 $\alpha_{1}$ 为 0.58 , 长程标度指数 $\alpha_{2}$ 为 $0.74 ; 2008$ 年分别为 0.54 和 $0.75 ; 2009$ 年分别为 0.56 和 0.72 。短程标度指数和长程 标度指数均具有较好的稳定性, 且每一年份的短程标 度指数 $\alpha_{1}$ 均小于长程标度指数 $\alpha_{2}$ 。 $\alpha_{1}$ 和 $\alpha_{2}$ 均在 $0.5 \sim 1.0$ 之间, 表明全市火灾的时间间隔序列具有稳定的长程 幂律相关性。 


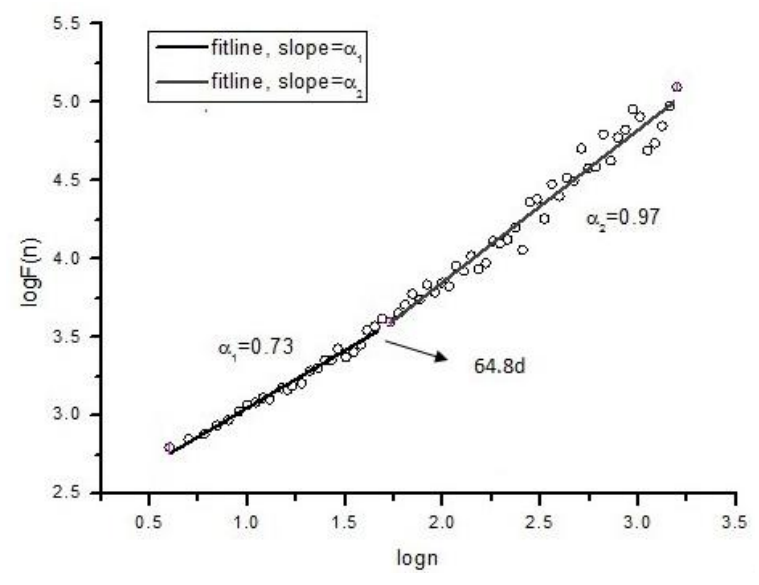

图4 2000-2009年全市火灾发生时间间隔序列的DFA 分析结果

表2. 2007-2009年合肥市每年火灾次数

\begin{tabular}{ll}
\hline Year & No. of fires \\
\hline 2007 & 1203 \\
2008 & 1458 \\
2009 & 1375 \\
\hline
\end{tabular}

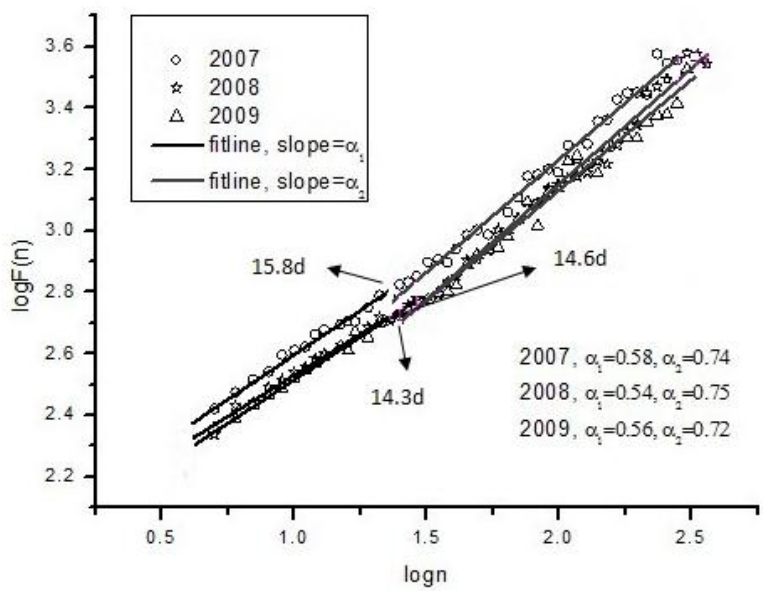

图5 单个年份全市火灾发生时间间隔序列的DFA分 析结果

图4和图5的结果显示，对于城市火灾发生时间 间隔, 在整个分割区间上存在交叉点, 即图中箭头 所指处，这说明城市火灾时间序列存在两个不同数 值的标度指数。某个系统的标度指数 $\alpha$ 并不总是常
数, 经常会随着不同的标度范围而改变, 即出现交 叉点。其原因通常认为是由于在不同时间和空间标 度下, 信号的相关性质发生改变而引起的, 反映不 同的物理含义。但在本文中, 在整个分割区间内城 市火灾发生时间间隔序列的两个标度指数的数值都 在0.5 1之间, 是同一性质的标度指数, 只是强弱程 度的区别。这说明城市火灾发生时间的序列具有长 期记忆特征, 当前发生的城市火灾和未来火灾事件 之间存在长期相关性, 从而在时间尺度上根据现在 及以前的城市火灾对未来的城市火灾进行预测是科 学可行的, 且所分析的时间区间越长, 结果会越合 理。

3.2 具有不同社会经济特征的城市区域火灾数据的 DFA分析结果（较小空间尺度）

一个城市往往具有不同的功能区域，对于基于社 区的防火概念来说, 需要找出不同城市区域中火灾发 生的特征，而不仅仅是关注于整个城市的火灾数据。 因此，本文将DFA方法进一步应用于分析2000-2009 年合肥市的六个不同辖区内的火灾时间序列, 结果 如图6所示。发现这六个辖区的火灾分布与全市的 火灾分布具有一致的形式, 即每个辖区内的火灾发 生时间间隔序列均表现出一种稳定的长程相关性, 且具有分段的标度指数特征, 分段处的位置分别 为: 蜀山区 23 天, 庐阳区 32 天, 包河区 38 天, 瑶海 区 25 天，高新技术产业区 16 天，经济开发区 13 天， 均小于图4中全市火灾时间序列分段点处的分割时 间值，64.8天，这说明城市火灾的空间背景越大， 对其发生时间进行分析和预测时所需要的过去的连 续事件数越多。对于这四个中心城区, 短程标度指 数 $\alpha 1$ 数值在 0.65 至 0.72 , 与全市火灾发生时间间隔 序列的短程标度指数 0.73 十分接近, 而长程标度指 数 $\alpha 2$ 数值在 0.81 至 0.91 , 比全市火灾序列的长程标 度指数 0.97 稍小。对于两个新近设立的开发区, 短程 标度指数与长程标度指数比较接近, 且短程标度指 数均明显高于四个中心城区, 而长程标度指数与四 个中心城区及全市的火灾序列比较类似, 均接近于 1.0, 差距不大。 
四个中心城区即包河区、蜀山区、庐阳区和瑶 海区几乎包括了合肥市 $90 \%$ 的居住人口, 以及全市的 大部分商业活动、社会活动和传统工业活动, 而高 新技术产业区和经济开发区主要通过一些高科技产 业来促进合肥经济的发展, 在这两个区域中无论是 人口还是传统的社会活动, 都大大少于四个中心城 区。结合四个中心城区与两个开发区在短程标度指 数上的明显差别, 可以认为与人口和社会经济因素 密切相关的人类活动对城市火灾在较短时间尺度上 的动态特征会造成影响。因此, 在这两个开发区 中, 能在较短时间尺度上体现出来的人口与社会经 济因素对城市火灾分布的影响并不明显, 从而短程 标度指数 $\alpha 1$ 与长程标度指数 $\alpha 2$ 在数值上更为接近, 亦即在这两个区域内, 无论是从较短还是较长时间 尺度下进行分析, 其城市火灾的动态发展是类似 的。然而, 对于四个中心城区来说, 短程标度指数 $\alpha 1$ 明显小于长程标度指数 $\alpha 2$, 这就意味着在这四个 区域中, 当从较短时间尺度进行分析时, 城市火灾 的分布形式与人口及社会经济方面的因素具有比较 强的内在联系。当从较长时间尺度进行分析时, 全 部六个区域均表现出稳定的长程相关性, 长程标度 指数接近于 1.0 , 这就意味着在长时间尺度上, 城市 火灾系统的动态特征与人类活动关系不大, 而是主 要受到一些与城市的普遍特征如城市结构、气候等 因素有关的内在机制的控制。

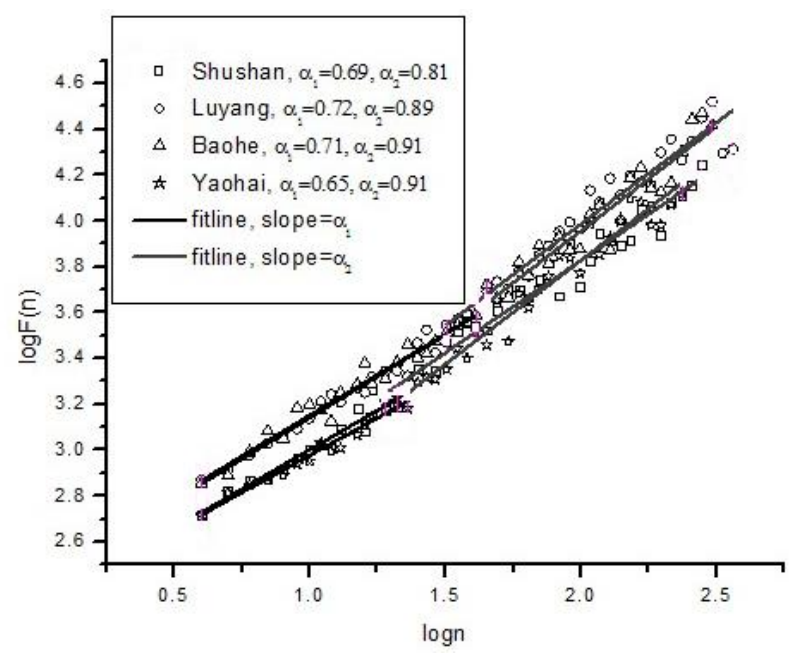

(a)

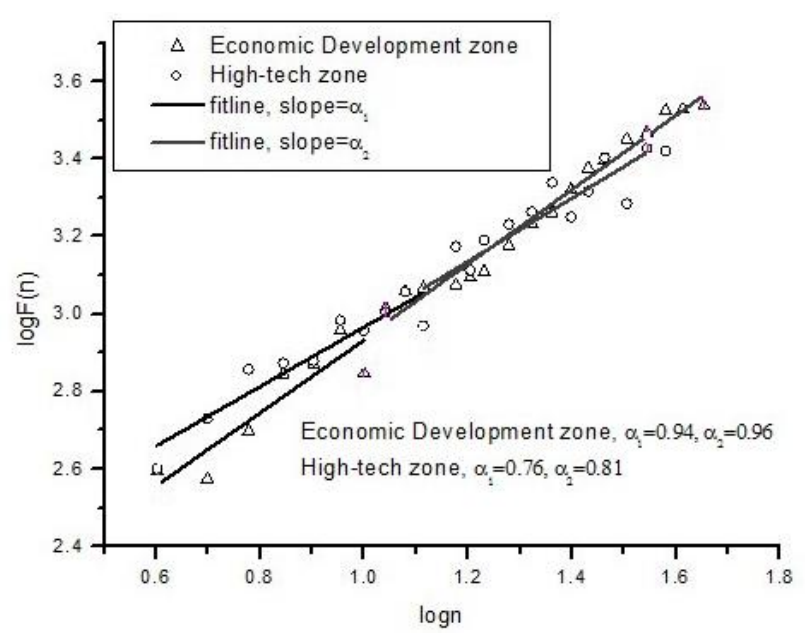

(b)

图 6 2000-2009 年不同区域城市火灾发生时间间隔 序列的 DFA 分析结果

\section{4 结论}

本文运用去趋势波动分析方法, 在不同的空间尺 度上对一个典型的中国发展中城市合肥的火灾发生及 损失规模时间序列的长程相关性特征进行了分析, 发 现城市火灾造成的直接经济损失时间序列在长程时间 尺度上表现出稳定的反幂律相关性, 即所分析序列具 有非持久性, 过去发生的事件对未来事件具有不确定 的影响。损失规模一定程度上反映了火灾在空间上的 分布特征, 而这种空间上的长程特征应是受到某种动 力学机制的作用, 即与时间上的分布形式有所联系。 进一步的研究发现, 城市火灾在时间上确实具有长程 相关性的特征。通过对比研究全市及不同城市区域的 火灾序列, 发现无论是在全市的大空间尺度范围或是 某个区域的小空间尺度范围内, 城市火灾发生的时间 间隔序列都表现出稳定的长程幂律相关性, 且具有分 段的标度指数特征, 短程标度指数 $\alpha 1$ 与长程标度指数 $\alpha 2$ 均位于 0.5 至 1.0 之间。

结合这种分段标度指数的特征, 本文进一步讨论 了城市火灾系统的内在动力学机制。对于两个经济开 发区来说, 短程标度指数 $\alpha 1$ 明显高于四个中心城区, 而长程标度指数 $\alpha 2$ 与四个中心城区及全市相差不大, 均接近于1.0。由于四个中心城区与两个经济开发区之 间的主要差异在于人口规模及社会经济活动等方面, 
因此本文认为人类活动对城市火灾系统的动态影响主 要普遍存在于较短的时间尺度上, 而在较长的时间尺 度上, 人类活动对城市火灾系统的这种长程相关性特 征并无明显的影响。在较长时间尺度上, 城市火灾系 统的动态特征主要受到一些与城市的普遍特征如城市 结构、气候等因素有关的内在机制的控制。

\section{致谢}

本文受中国香港特别行政区研究委员会项目基金资助

（项目号：CityU118708），在此表示感谢。

另: 本文的原始英文版本已发表在第三届风险分析与 危机响应国际会议论文集。

\section{参考文献}

[1] Bak P, Tang C, Wiesenfeld K. Self-organized criticality: an explanation of $1 / \mathrm{f}$ noise. Phys Rev Lett, 1987, 59: 381-384

[2] Hwa T, Kardar M. Fractals and self-organized criticality in dissipative dynamics. Phys D, 1989, 38(1-3): 198-202

[3] Song W G, Wang Q, Wang J. Characteristics of fire distribution in Huludao City and its enlightenment to city fire danger analysis. Sci \& Technol Rev, 2005, 23(8): 35-37

[4] Wang J, Song W G, Zheng H Y. Temporal scaling behavior of human-caused fires and their connection to relative humidity of the atmosphere. Ecol Model, 2010,221(1):85-89

[5] Guo T N, Fu Zh M. The fire situation and progress in fire safety science and technology in China[J]. Fire Safety J, 2007,42:171-182.

[6] J Alvarez-Ramirez, R Escarela-Perez, G Espinosa-Perez, et al. Dynamics of electricity market correlations. Phys A, 2009,388:2173-2188.

[7] Peng C K, Buldyrev S V, Havlin S, et al. Mosaic organization of DNA nucleotides. Phys Rev E, 1994,49:1685-1689.

[8] Nagarajan R, Upreti M, Govindan R B. Qualitative assessment of cDNA microarray gene expression data using detrended fluctuation analysis. Phys A, 2007,373:503-510.

[9] Perakakis P, Taylor M, Martinez-N E, et al. Breathing frequency bias in fractal analysis of heart rate variability. Biol Psychol, 2009,82(1): 82-88

[10] Stubsjøen S M, Bohlin J, Skjerve E, et al. Applying fractal analysis to heart rate time series of sheep experiencing pain. Physiology \& Behavior, 2010,101(1): 74-80

[11] Koçak K. Examination of persistence properties of wind speed records using detrended fluctuation analysis. Energy, 2009,34(11):1980-1985

[12] Yuan N M, Fu Z T, Mao J Y. Different scaling behaviors in daily temperature records over China. Phys A, 2010,389(19): 4087-4095

[13] Yudong Wang, Li Liu, Rongbao Gu, et al. Analysis of market efficiency for the Shanghai stock market over time. Phys A, 2010,389(8):1635-1642

[14] Rongbao Gu, Hongtao Chen, Yudong Wang. Multifractal analysis on international crude oil markets based on the multifractal detrended fluctuation analysis. Phys A, 2010,389(14): 2805-2815

[15] L. Telesca, Lovallo M, Lapenna V, et al., Long-range correlations in two-dimensional spatio-temporal seismic fluctuations, Phys A, 2007,377:279-284.

[16] Hongyang Zheng, Weiguo Song, Jian Wang. Detrended fluctuation analysis of forest fires and related weather parameters. Phys A, 2008, 387 :2091-2099

[17] Yue J H, Zhao X J, Shang P J. Effect of Trends on Detrended Fluctuation Analysis of Precipitation Series. Math Probl Eng,2010:1-15. 